Original Article

\title{
Freeze-dried equine-derived redback spider antivenom: a local irritation study by intramuscular injection in rabbits and a repeated-dose toxicity study in rats
}

\author{
Akihiko Yamamoto1*, Satomi Harano², Noriko Shinya ${ }^{2}$, Ayataka Nagano², \\ Yoshinobu Miyatsu ${ }^{2}$, Kyouko Sawabe 3 , Takayuki Matsumura ${ }^{4}$, Manabu Ato ${ }^{4}$, \\ Motohide Takahashis, Hisashi Taki ${ }^{6}$, and Toru Hifumi ${ }^{7}$ \\ ${ }^{1}$ Division of Biosafety Control and Research, National Institute of Infectious Diseases, 4-7-1 Gakuen, Musashimurayama-shi, Tokyo \\ 208-0011, Japan \\ ${ }_{2}^{2}$ The Chemo-Sero-Therapeutic Research Institute (KAKETSUKEN), 1-6-1 Okubo, Kita-ku, Kumamoto-shi, Kumamoto 860-8568, \\ Japan \\ ${ }^{3}$ Department of Medical Entomology, National Institute of Infectious Diseases, 1-23-1 Toyama, Shinjuku-ku, Tokyo 162-8640, Japan \\ ${ }_{4}^{4}$ Department of Immunology, National Institute of Infectious Diseases, 1-23-1 Toyama, Shinjuku-ku, Tokyo 162-8640, Japan \\ 5 Pharmaceuticals and Medical Devices Agency, 3-3-2 Kasumigaseki, Chiyoda-ku, Tokyo 100-0013 Japan \\ ${ }^{6}$ Ministry of Health, Labour and Welfare, Tokyo 100-8916, Japan \\ ${ }^{7}$ Emergency Medical Center, Kagawa University Hospital, 1750-1 Ikenobe, Miki, Kita-gun, Kagawa 761-0793, Japan
}

\begin{abstract}
The redback spider (Latrodectus hasseltii) is nonindigenous to Japan but has now spread throughout the country. Bites to humans are rare but can be fatal. We prepared freeze-dried redback spider antivenom for therapeutic use against bites in Japan by immunization of horse plasma. This study included two nonclinical tests of the antivenom: a local irritation study involving a single intramuscular administration to rabbits (with injections of physiological saline and an existing freeze-dried diphtheria antitoxin as control and comparison substances, respectively) and a 2-week repeated intermittent intravenous-dose toxicity study in rats. The irritation study showed the antivenom's irritancy to be comparable with that of the saline and the existing antitoxin preparations under the test conditions. In a repeated-dose toxicity study, no toxicity change was found in male or female rats, and the no-observed-adverseeffect level (NOAEL) was judged to be a dose volume of $20 \mathrm{~mL} / \mathrm{kg}$ (1082 units $/ \mathrm{kg}$ antivenom activity) in both male and female rats. In addition, there was no toxicological difference between proteinaceous diphtheria antitoxin and redback spider antivenom prepared to have the same protein content and the same additive composition. Based on these findings, we will further advance our research towards clinical application of the redback spider antivenom. This research was supported by the Research Program on Emerging and Re-emerging Infectious Disease of the Japan Agency for Medical Research and Development. (DOI: 10.1293/tox.2017-0053; J Toxicol Pathol 2018; 31: 105-112)
\end{abstract}

Key words: freeze-dried redback spider antivenom, equine polyclonal antibody, local irritation study, repeated-dose toxicity study

\section{Introduction}

Redback spiders (RBSs) (Latrodectus hasseltii) produce the venomous neurotoxin alpha-latrotoxin ${ }^{1}$. The RBS originated in Australia but has now been confirmed in New Zealand, various European countries, Southeast Asia, and the United States². In Japan, it was first reported in 1995 in

Received: 14 September 2017, Accepted: 19 December 2017 Published online in J-STAGE: 19 February 2018

*Corresponding author: A Yamamoto (e-mail: yama-aki@nih.go.jp) (C)2018 The Japanese Society of Toxicologic Pathology

This is an open-access article distributed under the terms of the Creative Commons Attribution Non-Commercial No Derivatives (by-nc-nd) License. (CC-BY-NC-ND 4.0: https
creativecommons.org/licenses/by-nc-nd/4.0/).
Takaishi City in Osaka Prefecture, Yokkaichi City in Mie Prefecture, and Miyakojima City in Okinawa Prefecture? ${ }^{3}$. RBSs were first sighted in Metropolitan Tokyo in 2014, and they are rapidly becoming a nationwide problem in Japan ${ }^{3}$. We believe that there is a risk they will spread throughout East Asia due to climate change and intensive interaction between people.

Most physicians in Japan are unfamiliar with RBS bites, as they are rare occurrences, so there is only limited clinical experience ${ }^{2}$. The symptoms of the bites are usually mild and localized, such as localized pain and erythema ${ }^{2}$. However, before the development of a specific RBS antivenom (RBSAV), which is manufactured through the immunization of horses, these bites often proved to be fatal ${ }^{4}$. RBSAV is produced by the Commonwealth Serum Laboratories (CSL) in Australia, but at present, it is considered an 
unapproved drug in Japan and therefore must be privately imported $^{2}$. This issue was further complicated by the suspension of RBSAV imports from CSL in autumn 2013. Japan's Ministry of Health, Labour and Welfare launched a research group to evaluate the safety and efficacy of the antivenom and to organize and maintain information on all RBS bites in April 20132. This group carefully evaluated the option of domestic RBSAV production for RBS bite emergencies. The first stage of production began in April 2014 and was completed in March 20165. The RBSAV developed in Japan differed from that prepared by CSL in that longterm preservation was possible for an agent type of the lyophilized product6.

Appropriate toxicity and efficacy studies were required prior to use the newly produced antivenom in clinical practice. As for the Japanese antitoxin preparation, to the old preparation of the development generation, before the modern requirements for testing and approval came into force; nonclinical studies of the earlier preparation had therefore not been conducted. In Japan, the toxicity of antivenom can be assessed by comparing it with that of already approved antivenom for a different antigen prepared in a similar way (in this case, from horse plasma).

The objective of this study was to perform nonclinical toxicity studies of RBSAV, specifically a local irritation study and an repeated-dose toxicity study. Because this RBSAV is generally administered intramuscularly, we conducted an intramuscular administration local irritation study using rabbits, which can receive the same dosage as humans. To allow the possibility of intravenously administering RBSAV, we conducted an intravenous study in rats to confirm the systemic toxicity. Because RBSAV is a preparation derived from horse plasma, it was necessary to consider serum disease.

\section{Materials and Methods}

\section{Local irritation study}

Animals: Twelve 15-week-old male rabbits weighing $2.55-2.94 \mathrm{~kg}$ at the initiation of the study were purchased from Kitayama Labes Co. Ltd. (Minowa, Japan). All the animals were housed individually in stainless steel cages kept in a room maintained at $21.9-23.8^{\circ} \mathrm{C}$, with $37-61 \%$ relative humidity and 12-h light and dark cycles. Solid feed (LRC4, Oriental Yeast Co., Ltd., Tokyo, Japan) was provided once daily to each animal. Water was available ad libitum from an automatic supply. The animals were grouped by stratified randomization of weight (MiTOX, Mitsui Zosen System Research Inc., Tokyo, Japan) to avoid differences in weight between the groups on the final day of acclimation. A local irritation study and repeated-dose toxicity study were approved by the Institutional Animal Care and Use Committee of Shin Nippon Biomedical Laboratories, Ltd., and were performed in compliance with domestic and international requirements, following good practice for animal welfare.

Sample and control materials: Freeze-dried equine-derived RBSAV, which contained 541 units of RBSAV activ- ity, was provided by the Chemo-Sero-Therapeutic Research Institute (KAKETSUKEN). To prepare the RBSAV for injection, $1 \mathrm{~mL}$ of physiological saline was added to each vial (541 units/mL: 1-fold dilution). It is stipulated that one unit of RBSAV is a unit of antitoxin that neutralizes $10 \mathrm{mg}$ of toxin?.

Physiological saline (Otsuka Pharmaceutical Co. Ltd., Tokushima, Japan) was used as the negative control, and the existing diphtheria antitoxin, provided by KAKETSUKEN, was used as the comparative control.

Treatment: Before administration, the fur on the right and left femurs was shaved with an electric hair clipper. A single injection of either the RBSAV or the existing diphtheria antitoxin was administered to the right side of the femoral muscle (musculus vastus lateralis or tensor fasciae latae muscle), and physiological saline was administered to the left femoral muscle. A disposable syringe, an extension tube, and a $23 \mathrm{G}$ needle with a plastic stopper were used for administration. The needle was inserted into the femoral muscle or musculus vastus lateralis vertically at a depth of approximately $1 \mathrm{~cm}$ at the center of the muscle. The local irritation of the muscle was examined on days 2 and 14 after administration by a method described later.

Observations and examinations: All animals were observed for clinical signs daily. Body weight was measured at the start and end of quarantine and at the end of acclimation. The observation of the injection site was performed before the injection and approximately 3 and $24 \mathrm{~h}$ after injection. Irritation at the injection site was evaluated according to the Draize method standards ${ }^{8}$.

Pathology: The body weights of the rabbits in each group ( 3 per group) were measured either 2 or 14 days after administration. The animals were then anesthetized by an auricular intravenous administration of an aqueous solution of pentobarbital sodium $(64.8 \mathrm{mg} / \mathrm{mL}, 0.7-0.9 \mathrm{~mL} /$ kg; Tokyo Chemical Industry Co., Ltd., Tokyo, Japan) and euthanized by exsanguination. The lateral vastus and tensor fasciae latae muscles and lesions around the muscles were sampled. When changes were observed on the muscle surface, the samples were sectioned longitudinally so as to include the most severe part of the externally observed abnormalities. When no change was observed on the muscle surface, the samples were sectioned longitudinally along the corresponding injection site. The sections were then subjected to macroscopic examination for hyperemia, bleeding, swelling, and white and brown discoloration evaluated on a scale of 0 (no abnormality) to 4 (marked change) (Table 1). For histopathological examination, tissue specimens of rabbit muscle tissue were fixed in $10 \%$ neutral buffered formalin solution. When lesions were observed macroscopically at an injection or corresponding site, the muscle fiber was sectioned transversally and laterally at 2 points, embedded in paraffin, and sectioned and stained with hematoxylin and eosin (HE). The specimens were observed for hemorrhage, edema, cell infiltration, degeneration, necrosis, and fibrosis in accordance with the criteria of histopathological evaluation ( 0 , no abnormal changes; 1 , very slight; 2 , slight; 3 , 
Table 1. Macroscopic Examination of Sectioned Muscle at the Injection Site in Male Rabbits 2 and 14 Days after Dosing (Local Irritation Study)

\begin{tabular}{|c|c|c|c|c|c|c|c|c|c|c|}
\hline \multirow[b]{2}{*}{ Group } & \multirow[b]{2}{*}{$\begin{array}{l}\text { Volume } \\
\text { (mL/site) }\end{array}$} & \multirow{2}{*}{$\begin{array}{c}\text { Days } \\
\text { after } \\
\text { dosing }\end{array}$} & \multirow[b]{2}{*}{ Animals } & \multirow[b]{2}{*}{ Site } & \multicolumn{6}{|c|}{ Score } \\
\hline & & & & & Hyperemia & Bleeding & Swelling & $\begin{array}{l}\text { White dis- } \\
\text { coloration }\end{array}$ & $\begin{array}{l}\text { Brown dis- } \\
\text { coloration }\end{array}$ & Total \\
\hline Physiological saline & 1.0 & 2 & 6 & Left & 0.0 & 0.2 & 0.0 & 0.0 & 0.0 & 0.2 \\
\hline Diphtheria antitoxin & 1.0 & 2 & 3 & Right & 0.0 & 0.0 & 0.0 & 0.0 & 0.0 & 0.0 \\
\hline Freeze-dried equine-derived RBSAV & 1.0 & 2 & 3 & Right & 0.0 & 0.0 & 0.0 & 0.0 & 0.0 & 0.0 \\
\hline Physiological saline & 1.0 & 14 & 6 & Left & 0.0 & 0.0 & 0.0 & 0.0 & 0.0 & 0.0 \\
\hline Freeze-dried equine-derived RBSAV & 1.0 & 14 & 3 & Right & 0.0 & 0.0 & 0.0 & 0.0 & 0.0 & 0.0 \\
\hline
\end{tabular}

Scores: 0 , no abnormal changes; 1 , very slight; 2 , slight; 3 , moderate; 4 , marked.

moderate; 4, marked). The most severe findings from two sections were selected per muscle.

\section{Repeated-dose toxicity study}

Animals: Forty 6-week-old male and forty 6-week-oldfemale Crl: CD(SD) rats weighing 159-241 g at the initiation of dosing were purchased from Charles River Laboratories Japan Inc. (Yokohama, Japan). All the animals were housed individually in stainless steel cages kept in a room maintained at $21.9-23.2^{\circ} \mathrm{C}$ with $43-57 \%$ relative humidity and 12-h light and dark cycles. Solid feed (CRF-1, Oriental Yeast Co., Ltd., Tokyo, Japan) was provided ad libitum to each animal. Water was available ad libitum from an automatic water supply system. The animals were assigned to groups by stratified randomization (MiTOX, Mitsui Zosen System Reseach Inc., Tokyo, Japan) according to body weight to minimize bias in body weight among the groups on the final day of acclimation.

Sample and control materials: RBSAV for the repeated-dose toxicity study was prepared in the same manner as for the local irritation study. The RBSAV and the diphtheria antitoxin were then diluted 10 -fold with physiological saline to achieve concentrations of 54.1 units $/ \mathrm{mL}$ and 50 units $/ \mathrm{mL}$.

Treatment: The rats were divided into four groups, each containing ten male and ten female rats. The two test article groups received 1 or $20 \mathrm{~mL} / \mathrm{kg}$ of the RBSAV, the control group received physiological saline, and the fourth group received $20 \mathrm{~mL} / \mathrm{kg}$ of the diphtheria antitoxin as a comparative substance. The test articles were administered into the tail vein of each rat using a disposable syringe, indwelling needle, extension tube, and infusion pump (BS-8000, Braintree Scientific Inc., Braintree, MA USA) at a rate of $0.5 \mathrm{~mL} / \mathrm{min}$. A second administration was performed after two weeks, i.e., on day 14 after the first injection on day 0 .

Observations and examinations: Clinical signs were observed once daily, and body weight and food consumption were measured once weekly. On the other hand, hematology and blood chemistry were performed at days 16 and 42 (at 2 and 28 days after the second administration) in 5 males and 5 females per group using blood collected via the abdominal aorta under anesthesia just before the animals were euthanized. The hematological examination included tests for the following red and white blood cell counts, hematocrit value, hemoglobin concentration, platelet count, mean corpuscular volume, mean corpuscular hemoglobin, mean corpuscular hemoglobin concentration, reticulocyte ratio, leukocyte count ratio, prothrombin time, activated partial thromboplastin time, and fibrinogen. They were measured using an ADVIA 120 Hematology System (Siemens, Munich, Germany) and Sysmex CA-7000 System (Sysmex, Corporation, Kobe, Japan). The blood chemistry included the following: aspartate transaminase, alanine transaminase, alkaline phosphatase, creatinine kinase, total bilirubin, total protein, albumin, globulin, total cholesterol, triglyceride, glucose, urea nitrogen, creatinine, inorganic phosphorus, calcium, sodium, potassium, chlorine, protein fraction (albumin ratio, alpha-1 globulin ratio, alpha-2 globulin ratio, beta globulin ratio, gamma globulin ratio and albumin/globulin), and Creactive protein, They were measured using a JCA-BM6070 automated biochemical analyzer (JEOL, Ltd., Tokyo, Japan) and an AES320 automated electrophoresis apparatus (Beckman Coulter Inc. USA).

For urinalysis, urine was collected from all the rats in each group within $4 \mathrm{~h}$ at days 15 and 41 using metabolic cages and subjected to urinalysis while still fresh. Color, $\mathrm{pH}$, protein, glucose, ketone bodies, bilirubin, occult blood, urobilinogen, and urine sediment were assessed using an automatic urine analyzer (Kimball Electronics Wales Ltd., Bridgend, UK). Urine sediments were examined for red blood cells, white blood cells, transitional epithelial cells, renal tubular epithelial cells, casts, and other findings after staining with Sternheimer-Malbin ${ }^{9}$. Urine volume, urine specific gravity, sodium, potassium, and the amount of chloride were measured collected $16 \mathrm{~h}$ preserved urine using.

Pathology: At days 16 and 42 (at 2 and 28 days after the second administration), 5 male and 5 female rats per group were weighed and anesthetized by intraperitoneal administration of an aqueous solution of pentobarbital sodium; blood samples were then collected for examination, and the animals were euthanized. The external surface, internal organs, and tissues of the animals were observed macroscopically. The histopathological examination included the weights of the lungs, submandibular glands, liver, heart, kidneys, testes, epididymides, prostate, seminal vesicles, ovaries, uterus, brain, spleen, thymus, pituitary, thyroid and adrenal glands; each of these were collected and weighed using an electronic balance (HR-200, A\&D Co., Ltd., Tokyo, Japan). The relative weights per $100 \mathrm{~g}$ were calculated 
from the body weight at necropsy. For the bilateral organs, the total left and right values were also calculated. The testes were fixed in formalin-sucrose-acetic acid solution, and other organs and tissues were fixed in $10 \%$ neutral buffered formalin solution. The injection site (the tail) was decalcified with Calcitox (Wako Pure Chemical Industries, Ltd., Osaka, Japan). For the high-dose (1,082 units/kg) and lowdose (54.1 unit $/ \mathrm{kg}$ ) RBSAV groups, saline group, and diphtheria antitoxin group, all organs and tissues such as the lungs, pancreas, liver, heart, kidneys, testes, epididymides, ovaries, uterus, brain, submandibular lymph nodes, mesenteric lymph nodes, spleen, thymus, adrenal glands, and tail were collected. After excision, the samples were embedded in paraffin, sliced, and stained with HE for histopathological examination. For the low-dose (54.1 unit $/ \mathrm{kg}$ ) RBSAV group, only the lungs, kidneys, and hearts were treated for histopathological examination.

Statistical analysis: Statistical analysis was performed for quantitative data, including body weight, food consumption, urinalysis data (quantitative data except electrolyte concentration), hematology data (excluding the differential leukocyte ratio), blood chemistry data, and organ weights (absolute and relative). First, the data from quantitative examinations were analyzed for homogeneity of variance using Bartlett's test ${ }^{10}$. When the variance was homogeneous, Dunnett's test ${ }^{11}$ was performed for multiple comparison between the control group and each test article group. When the variance was heterogeneous by Bartlett's test, a Dunnett-type test (Miller's test) was performed for multiple comparison between the control group and each test article group. In the diphtheria antitoxin group, the data from quantitative examinations were first analyzed for homogeneity of variance using by $F$ test. When the variance was homogeneous, Student's $t$ test was performed for comparison between the diphtheria antitoxin group and the saline control group. When the variance was heterogeneous by $\mathrm{F}$ test, the Aspin-Welch test ${ }^{12}$ was performed for comparison between the diphtheria antitoxin group and the saline control group. When this showed a significant difference, the data of the diphtheria antitoxin group and RBSAV groups were analyzed for homogeneity of variance using the F test. The Aspin-Welch test ${ }^{12}$ was performed for comparisons between the diphtheria antitoxin group and the RBSAV groups. When the variance was homogeneous, Student's $t$ test was performed for comparisons between the diphtheria antitoxin group and the RBSAV groups. When the variance was heterogeneous by F test, the Aspin-Welch test was performed for comparisons between the diphtheria antitoxin group and the RBSAV groups. For urinalysis, gradable data were analyzed by Wilcoxon's rank sum test ${ }^{13}$, and comparisons of urine color between the saline control group and the RBSAV groups or the diphtheria antitoxin group were performed using Fisher's exact test. The MiTOX System (Mitsui Zosen Systems Research Inc.) was used for these statistical analyses.

\section{Results}

\section{Local irritation study}

Observations and examinations: No animals died, and no abnormalities in clinical signs or body weight (detailed data are shown in supplementary Tables S1-1 and 2: online only) were observed in any animal during the observation period. Observation of the injection site showed no erythema/eschar or edema in any group. The results of the macroscopic examination are presented in Table 1. No abnormalities were observed in any case at the injection site of the RBSAV preparations at either 2 or 14 days after administration.

Pathology: The histopathological examination results are summarized in Table 2. At two days after administration of the RBSAV, very slight cell infiltration was observed at the injection site in one of the three animals. Hemorrhage, cell infiltration, and degeneration were observed at the injection site of the physiological saline, and the total mean score was 1.2. No abnormalities were observed at the injection site of the diphtheria antitoxin. At 14 days after administration, cell infiltration and degeneration were observed at the injection site of the RBSAV, and the total mean score was 1.0; cell infiltration and degeneration were also observed at the injection site of the diphtheria antitoxin, and the total mean score was 0.7. Degeneration was observed at the injection site of the physiological saline, and the total mean score was 1.0.

\section{Repeated-dose toxicity study}

Observations and examinations: No animals died during the observation period in any groups, and no test article-related changes were observed in clinical signs, body weight, food consumption, urinalysis, hematology, blood chemistry, necropsy, or weights of organs (detailed data are shown in supplementary Tables S2-1, S2-2, S2-3-1-4, S24-1, 2, S2-5-1, 2, S2-6, S2-7-1, and 2: online only). Table 3 summarizes some of the blood chemistry findings. The following significant differences in blood chemistry were found between the antivenom groups and the saline control group: at day 42, male rats in the high-dose RBSAV group had higher creatinine levels than those in the saline control group; male rats in the low-dose RBSAV group had higher C-reactive protein levels than those in the saline control group; female rats in the high-dose RBSAV group had lower sodium levels than those in the saline control group; and female rats in the low-dose RBSAV group had higher potassium levels than those in the saline control group. In addition, female rats in the high-dose RBSAV group had lower sodium levels than those in the diphtheria antitoxin group $(\mathrm{p}<0.01)$. However, these differences were judged not to be attributable to the RBSAV because no difference was dependent on the dose volume of RBSAV, and all individual values were within or slightly outside (in two animals only) the range of the background.

In addition, the C-reactive protein level was significantly higher in male rats in the diphtheria antitoxin group 
Table 2. Histopathological Findings at the Injection Site in Male Rabbits 2 and 14 Days after Dosing (Local Irritaion Study)

\begin{tabular}{|c|c|c|c|c|c|c|c|c|c|c|c|}
\hline \multirow[b]{2}{*}{ Group } & \multirow{2}{*}{$\begin{array}{l}\text { Volume } \\
\text { (mL/site) }\end{array}$} & \multirow{2}{*}{$\begin{array}{l}\text { Days } \\
\text { after } \\
\text { dosing }\end{array}$} & \multirow{2}{*}{ Animals } & \multirow[b]{2}{*}{ Site } & \multicolumn{7}{|c|}{ Score } \\
\hline & & & & & $\begin{array}{l}\text { Hemor- } \\
\text { rhage }\end{array}$ & Edema & $\begin{array}{c}\text { Cell } \\
\text { infiltration }\end{array}$ & $\begin{array}{l}\text { Degen- } \\
\text { eration }\end{array}$ & Necrosis & Fibrosis & Total \\
\hline Physiological saline & 1.0 & 2 & 6 & Left & 0.3 & 0.0 & 0.5 & 0.3 & 0.0 & 0.0 & 1.2 \\
\hline Diphtheria antitoxin & 1.0 & 2 & 3 & Right & 0.0 & 0.0 & 0.0 & 0.0 & 0.0 & 0.0 & 0.0 \\
\hline Freeze-dried equine-derived RBSAV & 1.0 & 2 & 3 & Right & 0.0 & 0.0 & 0.3 & 0.0 & 0.0 & 0.0 & 0.3 \\
\hline Physiological saline & 1.0 & 14 & 6 & Left & 0.0 & 0.0 & 0.0 & 1.0 & 0.0 & 0.0 & 1.0 \\
\hline Diphtheria antitoxin & 1.0 & 14 & 3 & Right & 0.0 & 0.0 & 0.3 & 0.3 & 0.0 & 0.0 & 0.7 \\
\hline Freeze-dried equine-derived RBSAV & 1.0 & 14 & 3 & Right & 0.0 & 0.0 & 0.7 & 0.3 & 0.0 & 0.0 & 1.0 \\
\hline
\end{tabular}

Scores: 0 , no abnormal changes; 1 , very slight; 2 , slight; 3 , moderate; 4 , marked.

Table 3. Blood Chemistry Findings in Rats Following Intravenous Injections of Redback Spider Antivenom, Diphtheria Antitoxin (for Comparison), or Saline (as the Control) (Repeated Dose Toxicity Study)

\begin{tabular}{lllccccc}
\hline \multirow{2}{*}{$\begin{array}{l}\text { Group } \\
\text { Dose }(\mathrm{mL} / \mathrm{kg})\end{array}$} & & & & Physiological saline & Diphtheria antitoxin & \multicolumn{2}{c}{ Freeze-dried equine-derived RBSAV } \\
\cline { 5 - 8 } $\mathrm{N}$ & & & & 20 & 20 & 1 & 20 \\
Creatinine & $(\mathrm{mg} / \mathrm{dL})$ & Male & Day16 & $0.234 \pm 0.029$ & $0.226 \pm 0.011$ & $0.232 \pm 0.035$ & $0.232 \pm 0.026$ \\
Creatinine & $(\mathrm{mg} / \mathrm{dL})$ & Male & Day42 & $0.272 \pm 0.023$ & $0.278 \pm 0.023$ & $0.304 \pm 0.032$ & $0.322 \pm 0.038^{*}$ \\
Creatinine & $(\mathrm{mg} / \mathrm{dL})$ & Female & Day16 & $0.264 \pm 0.019$ & $0.260 \pm 0.040$ & $0.246 \pm 0.021$ & $0.260 \pm 0.051$ \\
Creatinine & $(\mathrm{mg} / \mathrm{dL})$ & Female & Day42 & $0.320 \pm 0.023$ & $0.316 \pm 0.026$ & $0.324 \pm 0.011$ & $0.296 \pm 0.030$ \\
C-reactive protein & $(\mu \mathrm{g} / \mathrm{mL})$ & Male & Day16 & $731.144 \pm 87.947$ & $719.894 \pm 121.512$ & $868.284 \pm 80.511$ & $679.640 \pm 136.709$ \\
C-reactive protein & $(\mu \mathrm{g} / \mathrm{mL})$ & Male & Day42 & $675.102 \pm 17.909$ & $788.498 \pm 71.626^{\#}$ & $818.842 \pm 36.969^{*}$ & $742.786 \pm 159.534$ \\
C-reactive protein & $(\mu \mathrm{g} / \mathrm{mL})$ & Female & Day16 & $790.886 \pm 197.516$ & $829.644 \pm 79.287$ & $718.196 \pm 158.382$ & $858.990 \pm 59.511$ \\
C-reactive protein & $(\mu \mathrm{g} / \mathrm{mL})$ & Female & Day42 & $880.504 \pm 78.517$ & $847.386 \pm 93.848$ & $933.044 \pm 94.039$ & $977.170 \pm 182.411$ \\
Sodium & $(\mathrm{mEq} / \mathrm{L})$ & Male & Day16 & $142.8 \pm 1.1$ & $143.4 \pm 0.5$ & $143.0 \pm 0.7$ & $142.6 \pm 0.9$ \\
Sodium & $(\mathrm{mEq} / \mathrm{L})$ & Male & Day42 & $144.6 \pm 1.1$ & $144.0 \pm 1.0$ & $144.4 \pm 1.1$ & $144.0 \pm 0.7$ \\
Sodium & $(\mathrm{mEq} / \mathrm{L})$ & Female & Day16 & $142.8 \pm 0.8$ & $143.6 \pm 1.1$ & $143.2 \pm 0.8$ & $142.6 \pm 1.8$ \\
Sodium & $(\mathrm{mEq} / \mathrm{L})$ & Female & Day42 & $144.0 \pm 1.4$ & $143.4 \pm 0.5$ & $142.4 \pm 0.9$ & $142.0 \pm 0.7 * \dagger$ \\
Potassium & $(\mathrm{mEq} / \mathrm{L})$ & Male & Day16 & $4.08 \pm 0.18$ & $4.16 \pm 0.23$ & $4.14 \pm 0.37$ & $4.30 \pm 0.25$ \\
Potassium & $(\mathrm{mEq} / \mathrm{L})$ & Male & Day42 & $4.16 \pm 0.11$ & $4.16 \pm 0.11$ & $4.28 \pm 0.19$ & $3.96 \pm 0.23$ \\
Potassium & $(\mathrm{mEq} / \mathrm{L})$ & Female & Day16 & $3.84 \pm 0.30$ & $3.76 \pm 0.09$ & $3.92 \pm 0.13$ & $3.80 \pm 0.23$ \\
Potassium & $(\mathrm{mEq} / \mathrm{L})$ & Female & Day42 & $3.72 \pm 0.13$ & $3.82 \pm 0.30$ & $3.96 \pm 0.13^{*}$ & $3.82 \pm 0.11$ \\
\hline
\end{tabular}

Values are expressed as the mean $\pm \mathrm{SD} .{ }^{*} \mathrm{p}<0.05$ : Significant difference from physiological saline (vs. the freeze-dried $\mathrm{RBSAV}$ group). $\# \mathrm{p}<0.05$ : Significant difference from physiological saline (vs. the diphtheria antivenom group). ${ }^{\dagger} p<0.01$ : Significant difference from diphtheria antitoxin (vs. Freeze-dried RBSAV group).

than in those in the saline control group. However, this change was judged not to be an antitoxin-related change because it not accompanied by any other changes in the examinations, and all individual values were within range of the background.

Pathology: Tables 4 and 5 summarize the histopathological findings of the kidney at 2 and 28 days after the second administration of RBSAV, diphtheria antitoxin, or physiological saline (i.e., administration days 16 and 42) in male and female rats. The findings revealed very slight renal tubule hyaline droplets on both sides of the kidney on days 2 and 28 in the male rats in the RBSAV groups and on day 28 in the male rats in diphtheria antitoxin group. However, they were judged to be alpha $2 \mathrm{u}$-globulin, which is a finding specific to male rats and of relevance to humans ${ }^{14-16}$ because the hyaline droplets were only observed in male rats and were positive for Mallory-Heidenhain staining and negative for periodic acid-Schiff (PAS) staining. In addition, because no changes related to renal injuries considered to be caused by the RBSAV were observed in any examination, it was judged that these changes were not toxicologically significant.

The histopathological findings other than those of the kidney in the repeated-dose toxicity study for the RBSAV are summarized in supplementary Tables S2-8-1 and 2: online only. No test article-related histopathological changes were observed in any other organs.

\section{Discussion}

In this study, RBSAV, an equine-derived polyclonal antibody, was intramuscularly administered to rabbits in a local irritation study and was intravenously administered to rats in a repeated-dose toxicity study to evaluate its potential toxicity. The results showed that the irritancy of a single injection of the freeze-dried RBSAV into the muscle of rabbits was comparable to that of injections of physiological saline or the existing diphtheria antitoxin preparation under 
Table 4. Histopathological Findings in Rats 2 Days after Receiving the Second Dose in the Repeated-dose Toxicity Study

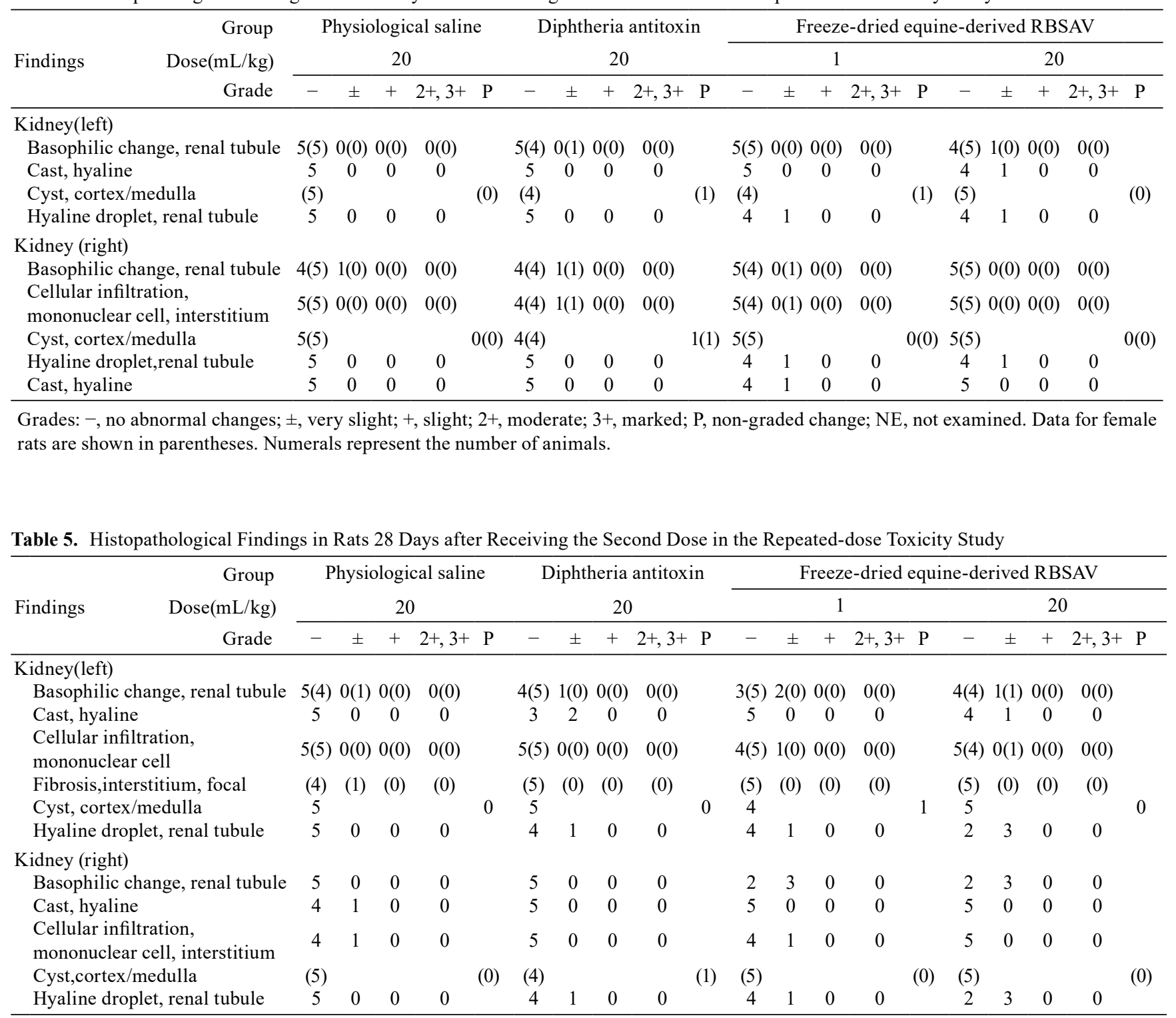

Grades: -, no abnormal changes; \pm , very slight; +, slight; 2+, moderate; $3+$, marked; P, non-graded change; NE, not examined. Data for female rats are shown in parentheses. Numerals represent the number of animals.

the same test conditions. The repeated-dose toxicity study showed no toxicity-related changes due to the RBSAV in male or female rats, and the no-observed-adverse-effect level (NOAEL) was judged to be a dose volume of $20 \mathrm{~mL} /$ $\mathrm{kg}(1,082$ units $/ \mathrm{kg}$ antivenom activity) in both sexes. In addition, there was no toxicological difference between the RBSAV and the freeze-dried proteinaceous diphtheria antitoxin prepared with the same protein content and the same additive composition as RBSAV under the conditions of these studies.

RBSAV was first developed as an approved drug by CSL in Australia about 60 years ago. There are very few published documents about the manufacturing process of this RBSAV. The World Health Organization (WHO) and other researchers have reported various methods for devel- oping snake antivenoms through horse immunization, but no consensus has yet been reached ${ }^{17-20}$. Only very limited information is available describing the immunization of mammals with spider venom, and relevant information on RBS venom was found only in a single report by Wiener $e t$ al. from 19617 .

We manufactured approximately 6,500 vials of a single-dose freeze-dried test lot using RBSs captured in Japan ${ }^{6}$. This test lot had a potency $(500 \mathrm{U} / \mathrm{vial})$ equivalent to the only commercial product in the world (bioCSL) along with a safety profile and physicochemical properties similar to those of existing antivenom drug products commercially available in Japan. Nonclinical toxicity studies, which are generally performed, were conducted to check the potential toxicity of the new RBSAV using rabbits and rats. 
Draize et al. ${ }^{8}$ developed a test for detecting local toxicity and irritation by using rabbits 70 years ago, and this method continues to be used as a local stimulation test for recently produced drugs $^{8}$. In the present local irritation study, no deaths were observed during the observation period, and no abnormalities were observed in clinical signs or body weight. In addition, no erythema/eschar formation or edema was observed at the injection site of the freeze-dried RBSAV, and a macroscopic examination found no abnormalities at the site at 2 and 14 days after injection. Furthermore, no abnormalities were found in a histopathological examination. No obvious irritancy was observed in the rabbits injected with RBSAV or the diphtheria antitoxin after a single intramuscular administration. These results suggest that RBSAV could be a safe and reliable formulation for clinical application.

The repeated-dose toxicity study was contrived to determine the dose level that may cause obvious toxic changes upon repeated administration of a test substance to mammals and the details of these changes, as well as the dose level at which no toxic changes are noted. No changes related to toxicity were observed in the clinical and histopathological examinations in the present study. Slight changes were observed in the clinical examination, but they were unrelated, and no differences were dependent on the dose volume; all individual values were within or slightly outside the normal range (in two animals only), it was therefore judged that they were not attributable to the RBSAV. In addition, serum sickness and anaphylaxis toxicity were not indicated, even with repeated administration of an enormous volume of 2 vials $/ \mathrm{kg}$ into the rat vein. All these findings suggest that, under the test conditions, the freeze-dried RBSAV caused no toxicity-related changes in male or female rats and that a dose volume of $20 \mathrm{~mL} / \mathrm{kg}$ (1,082 units $/ \mathrm{kg}$ protein dose) was a nontoxic dose for both male and female rats. In addition, there was no toxicological difference between the RBSAV and the diphtheria antitoxin prepared with the same protein content and the same additive composition as the RBSAV.

Compared with the popular equine-derived antivenom reported previously ${ }^{17-20}$, these studies showed that the RBSAV had relatively low toxicity and no adverse effects. Antivenom is the mainstay of treatment for snakebite envenoming, and adverse reactions to the available snake antivenom are common in many parts of the world where snakebites are prevalent. Both acute (anaphylactic or pyrogenic) and delayed (serum sickness type) reactions occur. Acute reactions are usually mild, but severe systemic anaphylaxis may develop, often within an hour or so of exposure to the antivenom. Serum sickness after antivenom has a delayed onset of between 5 and 14 days after administration. Ultimately, prevention of such reactions depends mainly on improving the quality of the antivenom. Until these improvements take place, doctors have to depend on pharmacological prophylaxis (with the search for the best prophylactic agent still in progress) as well as on careful observation of the patient receiving antivenom in readiness for prompt management of any acute or delayed reaction, should they occur ${ }^{21}$. Serum sickness is a delayed immune reaction resulting from the injection of foreign protein or serum. Antivenom is known to cause serum sickness, but the incidence and characteristics of serum sickness are poorly defined 22 .

Our RBSAV is consistent with $\mathrm{F}\left(\mathrm{ab}^{\prime}\right) 2$ equine-derived $\mathrm{IgG}^{6}$. Protein preparations made from heterologous proteins such as equine plasma have been reported to produce early and late side effects due to immune responses when administered to humans. The magnitude of the reaction depends on the content of the heterologous proteins contained in the preparation. According to the $\mathrm{WHO}$ antitoxin guidelines, it is recommended that the protein content be kept below 100 $\mathrm{mg} / \mathrm{mL}^{23}$. The RBSAV examined in this study has high specific activity because of successful immunization of horses in the manufacturing process 6 . This allowed the protein content of the RBSAV to be as low as $8.1 \mathrm{mg} / \mathrm{mL}^{6}$. It is suggested that the protein content being $1 / 12.5$ of the recommended value by the WHO guidelines is one of the reasons for the poor toxicity in rats.

The use of early antivenoms, such as the RBSAV produced by CSL, was associated with frequent acute adverse reactions and serum sickness. It is unclear what the risk of allergic reactions is with diluted intravenous CSL RBSAV when given appropriately. Ninety-five cases of the intravenous administration of CSL's RBSAV from January 2001 to November 2006 were reviewed ${ }^{24}$. All patients experienced local pain, $72 \%$ experienced radiating pain, $57 \%$ experienced diaphoresis, and 39\% experienced systemic effects. Four patients $(4 \%)$ experienced immediate systemic hypersensitivity reactions: none were severe, one was moderate, and three where mild. Of 32 patients followed up for 2 weeks, three (10\%) developed serum sickness. These results indicated that RBSAV given intravenously had a low reaction rate ${ }^{24}$. Passive immunization has been used in clinical practice since the late 1890s, mainly for prophylaxis. The success of early treatments was marred by anaphylactic reactions and serum sickness because antibodies or antitoxins were not raised in humans ${ }^{25}$.

We believe that our method of manufacturing RBSAV will be manufacturing an equine serum-derived medicine with a very low rate of appearance of associated reactions from now on because of these nonclinical studies. Therefore, after proper consideration of the manufacture of this RBSAV and deliberation by an ethics committee, clinical application in humans will be examined in a future study.

Disclosure of Potential Conflicts of Interest: The authors declare that they have no competing interests.

Acknowledgment: This research was supported by the Research Program on Emerging and Re-emerging Infectious Disease of the Japan Agency for Medical Research and Development (AMED). 


\section{References}

1. Graudins A, Padula M, Broady K, and Nicholson GM. Redback spider (Latrodectus hasselti) antivenom prevents the toxicity of widow spider venoms. Ann Emerg Med. 37: 154-160. 2001. [Medline] [CrossRef]

2. Aya Kumei YY, Imanishi H, and Nakagawa K. A case of Red Back spider envenomation. Jpn J Dermatol. 121: 18811884. 2011.

3. Japan 'not ready' for invasion of redbacks as venomous Australian spiders reach Tokyo. 2014, from ABC news website: http://www.abc.net.au/news/2014-10-20/redbackspiders-found-for-the-first-time-in-tokyo/5827612.

4. Braitberg G, and Segal L. Spider bites - Assessment and management. Aust Fam Physician. 38: 862-867. 2009. [Medline]

5. Hifumi T, Taki H, Yamamoto A, Ato M, Koido Y, and Kuroda Y. Update of antivenom supply for redback spider bites in Japan. J Intensive Care. 3: 7-8. 2015. [Medline] [CrossRef]

6. Mori S, Horita A, Ginnaga A, Miyatsu Y, Sawabe K, Matsumura T, Ato M, Yamamoto A, Shibayama K, Arai S, Yamagishi T, Takahashi M, Taki H, and Hifumi T. Venom and antivenom of redback spider (Latrodectus hasseltii) in Japan. II. Experimentally production of equine antivenom of redback spider. JJID. 70: 625-641. 2017.

7. Wiener S. Red back spider antivenene. Med J Aust. 48: 4144. 1961. [Medline]

8. Draize JH, Woodard G, and Calvery HO. Methods for the study of irritation and toxicity of substances applied topically to the skin and mucous membranes. J Pharmacol Exp Ther. 82: 377-390. 1944.

9. Goodgold AL, and Reubi F. Appraisal of the SternheimerMalbin urinary sediment strain in the diagnosis of pyelonephritis. Urol Int. 1: 225-242. 1955. [Medline] [CrossRef]

10. Bartlett MS. Properties of sufficiency and statistical tests. Proceedings of the royal society of London series A160, London, 268-282. 1937.

11. Dunnett CW. New-tables for mulyiple comparisons with a control. Biometrics. 20: 482-491. 1964. [CrossRef]

12. Welch BL. The generalisation of student's problems when several different population variances are involved. Biometrika. 34: 28-35. 1947. [Medline]

13. Wilcoxon F. Individual comparisons by ranking methods.
Biom Bull. 1: 80-83. 1945. [CrossRef]

14. Hard GC. Some aids to histological recognition of hyaline droplet nephropathy in ninety-day toxicity studies. Toxicol Pathol. 36: 1014-1017. 2008. [Medline] [CrossRef]

15. Peter G. Hyaline droplet. In: Histopathology of Preclinical Toxicity Studies. 3rd ed, Elsevier Science, Amsterdam, 600-601. 2007.

16. Frazier KS, Seely JC, Hard GC, Betton G, Burnett R, Nakatsuji S, Nishikawa A, Durchfeld-Meyer B, and Bube A. Proliferative and nonproliferative lesions of the rat and mouse urinary system. Toxicol Pathol. 40(Suppl): 14S-86S. 2012. [Medline] [CrossRef]

17. World Health Organization Guidelines for the production, Control and regulation of snake antivenom immunoglobulins 2010. http://www.who.int/biologicals/expert_committee/Antivenom_WHO_Guidelines_DJW_DEB_mn_cp.pdf.

18. Chippaux JP. Production and use of snake antivenom. In: Handbook of Natural Toxins Vol. 5 Reptile Venoms and Toxins, AT Tu (eds). CRC press, Ohio, 529-552. 1992.

19. Chotwiwatthanakun C, Pratanaphon R, Akesowan S, Sriprapat S, and Ratanabanangkoon K. Production of potent polyvalent antivenom against three elapid venoms using a low dose, low volume, multi-site immunization protocol. Toxicon. 39: 1487-1494. 2001. [Medline] [CrossRef]

20. Khomvilai S. Needs of antisera production centers. WHO consultative meeting on rabies and envenomings: A neglected public health issues, 2007. http://www.who.int/ snakebites/resources/9789241563482/en/.

21. de Silva HA, Ryan NM, and de Silva HJ. Adverse reactions to snake antivenom, and their prevention and treatment. $\mathrm{Br}$ J Clin Pharmacol. 81: 446-452. 2016. [Medline] [CrossRef]

22. Ryan NM, Kearney RT, Brown SG, and Isbister GK. Incidence of serum sickness after the administration of Australian snake antivenom (ASP-22). Clin Toxicol (Phila). 54: 27-33. 2016. [Medline] [CrossRef]

23. Minimum Requirements for Biological Products Ministry of health, labour and welfare. 2004. https://www.niid.go.jp/ niid/ja/mrbp.html.

24. Isbister GK. Safety of i.v. administration of redback spider antivenom. Intern Med J. 37: 820-822. 2007. [Medline] [CrossRef]

25. Llewelyn MB, Hawkins RE, and Russell SJ. Discovery of antibodies. BMJ. 305: 1269-1272. 1992. [Medline] [CrossRef] 